\title{
Appraise of Various Colour Spaces for Skin Detection based Background Removal from Hand Sign Images
}

\author{
Sudeep D. Thepade, PhD \\ Dean R\&D and Professor \\ PCCOE, Nigdi, Pune-44
}

\author{
Nilima Phatak, \\ Deepali Naglot \\ B.E Student \\ PCCOE Nigdi, Pune-44
}

\author{
Aishwarya Chandrasekaran, \\ Mugdha Joshi \\ B. E Student \\ PCCOE Nigdi, Pune-44
}

\begin{abstract}
Sign language is the basic medium of communication for the deaf and dumb people. It is a language which uses manual communication and body language to convey meaning. This can involve combining hand shapes, orientation and movement of hands. Communication may be the biggest challenge for the deaf and dumb in order to receive and convey information, ideas and feelings. Thus, in order to bridge the gap between them and the others, it becomes necessary to build a communicator and translator to translate American Sign Language to Indian and vice versa. In addition to this, the American and Indian sign language is also converted to text and back. During this translation, in order to ensure efficient skin detection and further processing of image, the paper focuses on obtaining appropriate results on Indian sign images based on background removal algorithms.
\end{abstract}

This paper presents the comparison among various color spaces for skin detection based on background removal from hand sign images. The color space is a useful way to specify and conceptualize the color capabilities of a particular digital file or an image. The proposed techniques on colour spaces are executed on a dataset of 78 images. In order to analyze the results of the image based on various color spaces, this study of comparison among them is needed. It elaborates mainly on four color models : RGB, YCbCr, HSV, NTSC. This paper analyses the results of the above color spaces.

\section{Keywords}

Color space, RGB(Red Green Blue), HSV(Hue Saturation Value),NTSC, YCbCr.

\section{INTRODUCTION}

To build an application for the deaf and dumb community has become of utmost importance today. With the changing world in terms of technology innovations and education opening new arenas of opportunities, it becomes necessary for everyone to be ahead of the others in this race. In order to enable the deaf and dumb community to create a recognition and also to give them a standard platform to communicate and express their opinions with every other individual, this application is being created.[2]

A sign language uses hand gestures in order to communicate with the other end. On the other end, it could be either a deaf and dumb person wishing to communicate or a normal person. With an initiative to build a unified interface between the two, this paper focuses at the essential preprocessing step needed to the system. The application would work as a translator to translate American sign language to Indian and vice versa. It would also convert text to either of the sign languages and back.
This would make communication among all people easier and convenient.

American Sign Language, considered to be one of the predominant sign language for deaf communities in United States, has become the widely used language all throughout the world. It uses only one hand to display the gestures and thus makes it easy for interpretation and understanding.

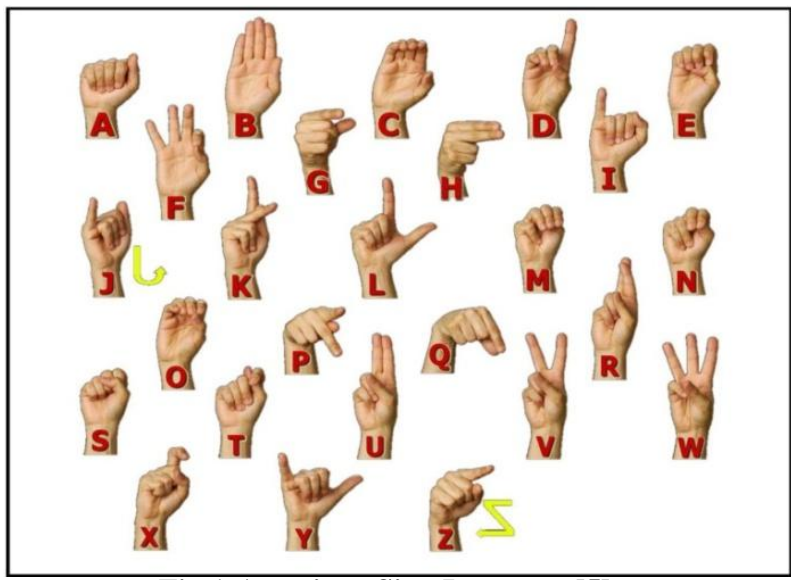

Fig 1-American Sign Language[5]

In this paper, the fundamental experimentation is done on Indian sign language. Indian Sign language is a sign language which uses both the hand gestures rather than the voice. ISL comprises of around 3000 gestures and other common words. The common words are shown with some specific gesture or spelling with the help of 26 hand gestures indicating 26 alphabets of ISL. This application initiates to focus on the importance to be given to ISL neglecting the limitations widely known.

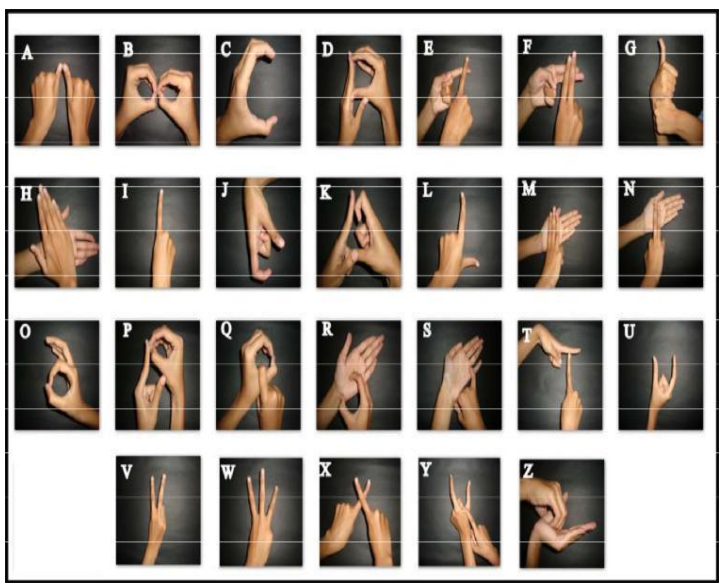

Fig 2-Indian Sign Language [6] 


\section{SKIN DETECTION}

Skin color has been a very useful hint for hand detection, localization and analysis of images in translation. Thus, there are various techniques which have been proposed for detection of skin pixels. The best approach is to build a skin classifier to specify the boundaries of skin cluster in color space.[3][4]

$(R, G, B)$ is classified as skin if:

$R>95$ and $G>40$ and $B>20$ and

$|R-G|>15$

(1) $\max \{R, G, B\}-\min \{R, G, B\}>15$ and

and $R>g$ and $R>B$

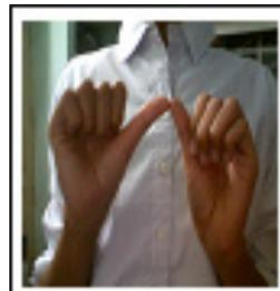

Original image

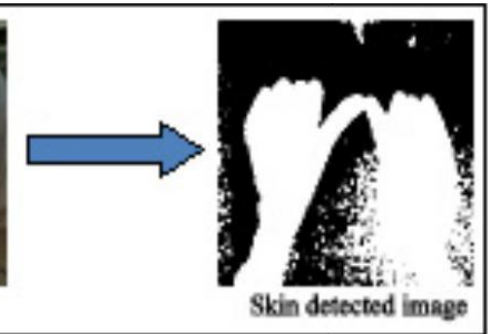

Fig 3: Skin detected image

\section{BASE BACKGROUND REMOVAL ALGORITHM}

Capture Sign image with Background(Image A) A sign image is captured using webcam having non-uniform background. The image referred as Image A acts as an original input image.

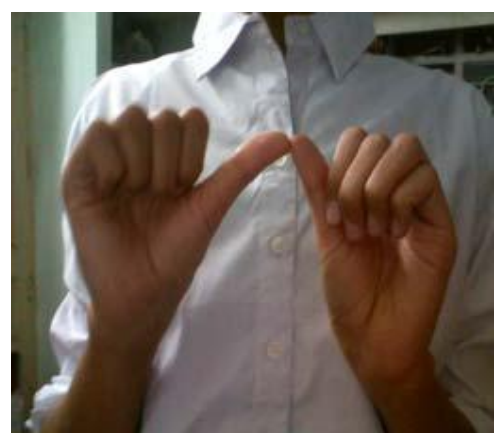

Fig 4: Image A

Skin Detection(Image B)

After capturing sign image, the skin region is captured using (1). The result obtained is shown in Fig 5 named as Image B.

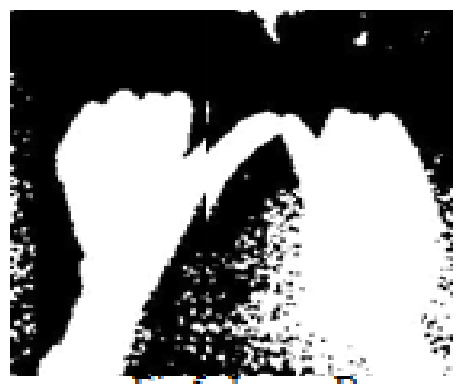

Fig 5: Image B
Morphological operation performed on skin detected image(Image C)

Morphological operations are performed on the Image B. Dilation operation is performed followed by Erosion operation. The result obtained is shown in Fig 6 and is named as Image C.

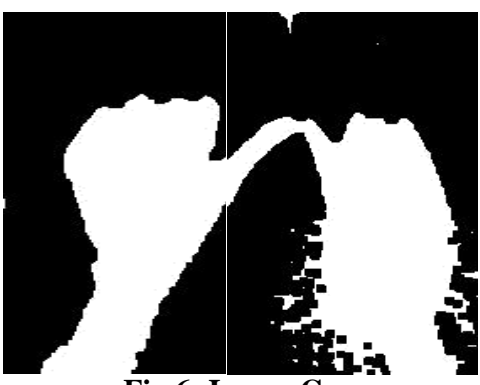

Edge detection on original input image with background(Image D)

Edge detection is performed using the Sobel Gradient operator on Image A to detect the edges. The result obtained is shown in Fig 7 and is named as Image D.

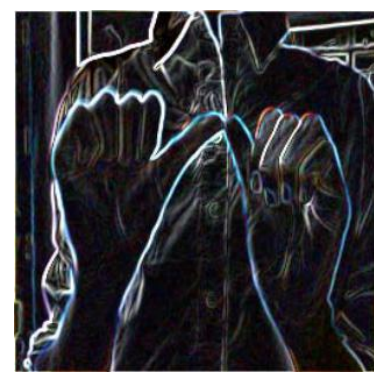

Fig 7: Image D

Superimposition of Image D and Image C (Image E) Image $\mathrm{C}$ and Image $\mathrm{D}$ are superimposed to remove the black patches, if any are present, as seen in Image C. The result obtained is shown in Fig 8 and is named as Image $\mathrm{E}$.

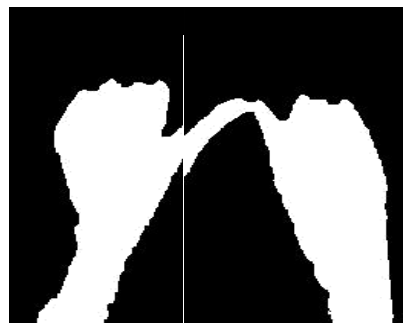

Fig 8: Image E

Superimposition of Image $\mathbf{E}$ and Image A(Resultant image) Image $\mathrm{E}$ is superimposed with the Image A to get the coloured image with nullified background. The result obtained is shown in Fig 9.

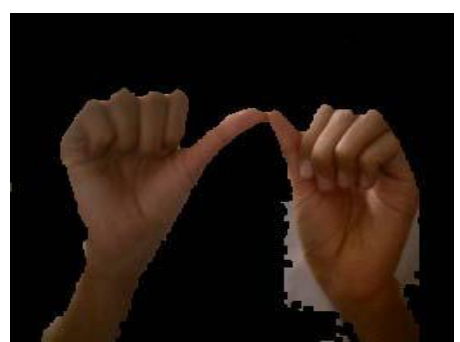

Fig 9: Resultant image 


\section{ALGORITHM:}

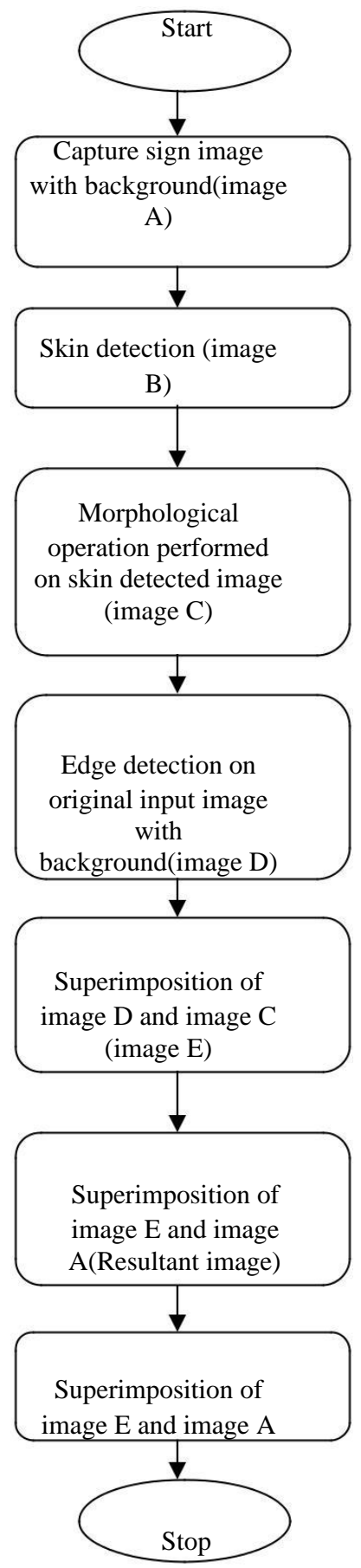

Fig 10: Background Removal Algorithm[1]

\section{COLOR SPACES}

Colour spaces provide a standard way to specify a particular colour, by defining a 3-D co-ordintate system and a subspace that contains all colours within a particular model. Any colour that can be specifies using a model will correspond to a single point within the subspace it defines. Each colour model is oriented towards specific colour spaces: RGB, YCbCr, HSV, NTSC.[7][8][11]

\subsection{RGB}

An RGB [13] image is stored as an m- by-n-by-3 data array that defines red,green,blue components for each individual pixel. The color of each pixel is determined by the combination of the red,green, blue intensities stored in each color plane at the pixel's location. Graphics file formats store RGB images as 24bit-images where the red, green and blue components are 8 bits each.

In an RGB array of class double, each color component is a value between 0 and 1 . A pixel whose color components are $(0,0,0)$ is displayed as black, and a pixel whose color components are $(255,255,255)$ is displayed as white. The three color components for each pixel are stored along the third dimension of the data array.

For example, the red, green, and blue color components of the pixel $(10,5)$ are stored in $\operatorname{RGB}(10,5,1), \operatorname{RGB}(10,5,2)$, and $\operatorname{RGB}(10,5,3)$, respectively.

RGB color space deals with the problem of varying illumination and the filter for uniform daylight illumination is:

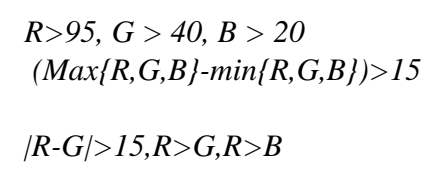

\section{2 $\mathrm{YCbCr}$}

$\mathrm{YCbCr}$ is a primary color space used to represent digital component video.

In $\mathrm{YCbCr}, \mathrm{Y}$ is the brightness(luma), $\mathrm{Cb}$ is blue minus luma(B$\mathrm{Y})$, Cr is red minus luma(R-Y). [9]

$$
\left[\begin{array}{c}
Y \\
C b \\
C r
\end{array}\right]=\left[\begin{array}{c}
16 \\
128 \\
128
\end{array}\right]+1 / 256\left[\begin{array}{ccc}
65.738 & 129.057 & 25.046 \\
-37.945 & -74.494 & 112.439 \\
112.439 & -94.154 & -18.285
\end{array}\right]
$$

The range at which skin pixels get detected are:

$$
\begin{gathered}
177>C r>137 \\
127>C b>77 \\
215>C b+0.6 C v>190
\end{gathered}
$$

\subsection{HSV}

The range at which skin pixels get detected are:

$H>=0 \& H<=0.25 \& S>=0.07 \& S<=0.9$

HSV is returned as an m-by-n-by-3 image array whose three planes contain the hue, saturation, and value components for the image.[12]

\subsection{NTSC}

NTSC is one of the widely used color space which realizes a clear separation between luminance and chrominance information. The components of NTSC color space are Y(the luminance component), I(the cyan-orange component), Q(the green-purple component).

In the NTSC color space, the luminance is the grayscale signal used to display pictures on monochrome (black and white) televisions. The other components carry the hue and saturation information.[10] 
The matrix for NTSC is:

$$
\left[\begin{array}{l}
Y \\
I \\
Q
\end{array}\right]=\left[\begin{array}{ccc}
0.299 & 0.587 & 0.114 \\
0.596 & -0.274 & -0.322 \\
0.211 & -0.523 & 0.312
\end{array}\right]\left[\begin{array}{l}
R \\
G \\
B
\end{array}\right]
$$

The ranges used for skin detection in NTSC are as follows:

$$
\begin{gathered}
N>=0.3 \& N<=0.9 \& \\
T>=0.05 \& T<=0.4 \& \\
S C>=(-1.9) \& S C<=1.0
\end{gathered}
$$

\section{IMPLEMENTATION}

\section{A. Platform Specifications}

The system is implemented in MATLAB version 12.0. The testing and training were run on standard laptop $(1.18 \mathrm{GHz}$ Core2Duo processor, 1.96GB of RAM running under windows XP) and web camera $(640 * 480$ resolution) is used for capturing images.

\section{B. Testbed Specifications}

The dataset used for training and testing is specified to consist of $78(26 * 3)$ sign images each of black and dark green background. Three signs of each alphabet are taken from 4 different volunteers. Each image is resized to $256 * 256$.

\section{Testing on proposed techniques}

Based on the observations obtained, we have classified the output on the basis of its results as excellent, good and satisfactory. The following table shows the analysis of all the color spaces and their results.

Table 1: Analysis of color spaces

\begin{tabular}{|c|c|c|c|c|}
\hline $\begin{array}{c}\text { Sr. } \\
\text { No }\end{array}$ & $\begin{array}{c}\text { Color } \\
\text { Space }\end{array}$ & Excellent & Good & Satisfactory \\
\hline 1. & RGB & Black & - & Green \\
\hline 2. & HSV & - & Black & Green \\
\hline 3. & YCbCr & - & Green & Black \\
\hline 4. & NTSC & Black & - & Green \\
\hline
\end{tabular}

The experimentation is performed on black and green backgrounds with four color spaces namely, RGB, HSV, YCbCr and NTSC.

The original image has been shown as Fig A in the below representation and it follows with the results of that image on the mentioned color spaces.

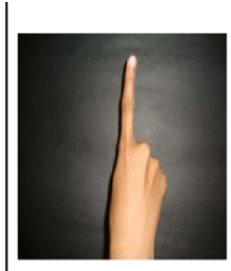

Fig A

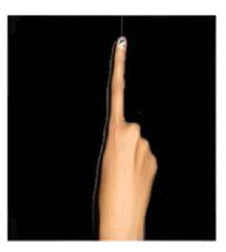

Fig A:1

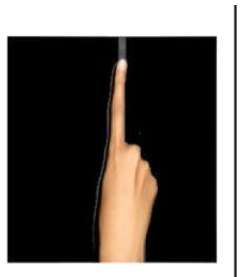

Fig A:2

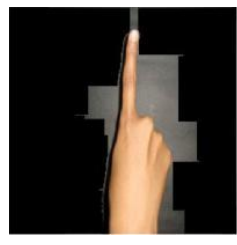

Fig A:3

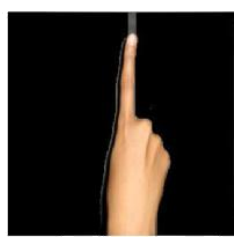

Fig A:4
The Fig A:1 shows the output of original image with RGB color space, Fig A:2 with HSV color space, Fig A:3 with YcbCr and Fig A:4 with NTSC color space. Observation shows that black background works best with RGB color space and gives poor results with $\mathrm{YCbCr}$.

The original image has been shown as Fig B in the below representation and it follows with the results of that image on the mentioned color spaces.

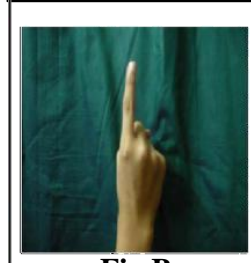

Fig B

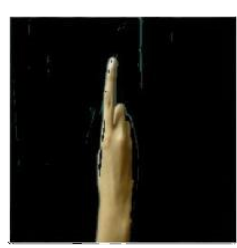

Fig B:1

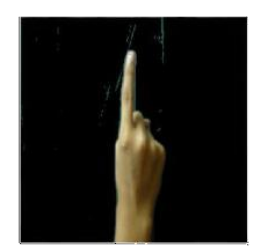

Fig B:2

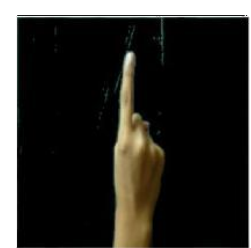

Fig B:3

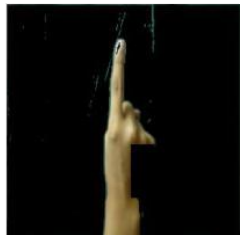

Fig B:4
The Fig B:1 shows the output of original image with RGB color space, Fig B:2 with HSV color space, Fig B:3 with YcbCr and Fig B:4 with NTSC color space. Observation shows that black background works well with $\mathrm{HSV}$ and $\mathrm{YCbCr}$ color spaces and gives poor results with NTSC.

In order to observe generalized results, the above mentioned color spaces have been applied on sample images. In Fig 11, 12 and 13, Fig (i) represents the original image on which the color spaces are applied. Fig (ii) represents the ideal output which is expected. Fig (iii), (iv), (v) and (vi) show the output of RGB, $\mathrm{HSV}, \mathrm{YCbCr}$ and NTSC color space. 


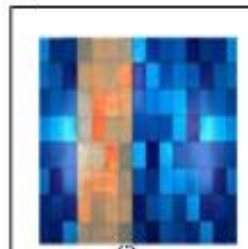

(i)

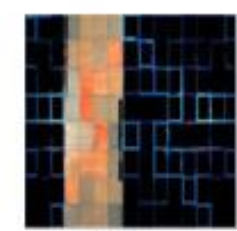

(iv)

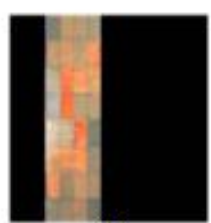

(ii)

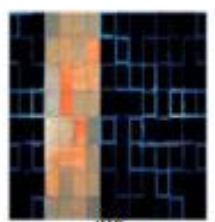

(iii)

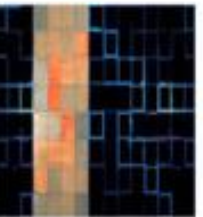

(v)

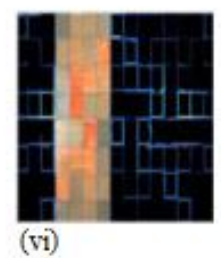

Fig.11

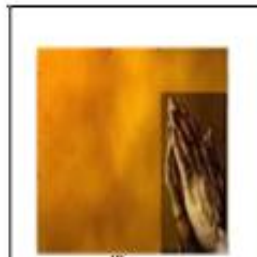

(1)

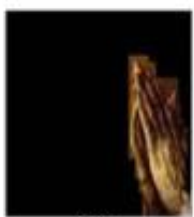

(11)

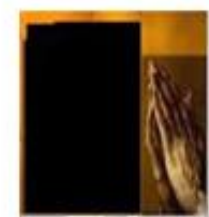

(iv)

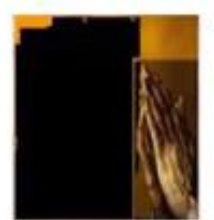

(v)

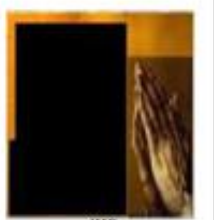

(iii)

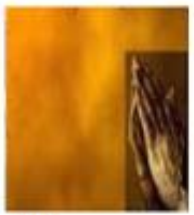

(vi)

Fig.12

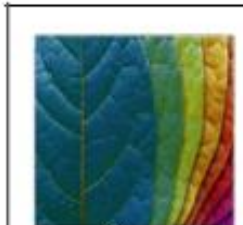

(1)

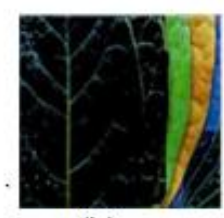

(iv)

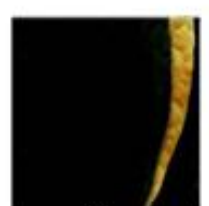

(ii)

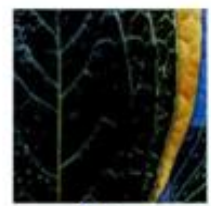

(v)

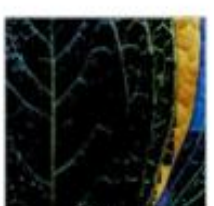

(iii)

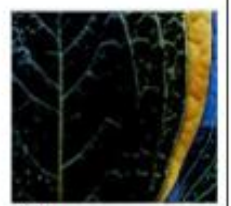

(vi)
Fig :13

\section{DISCUSSIONS AND CONCLUSION}

The proposed technique is tested on a dataset of 156 images 6 of each alphabet. Comparison of all the results using background removal approach is represented in Fig.14.

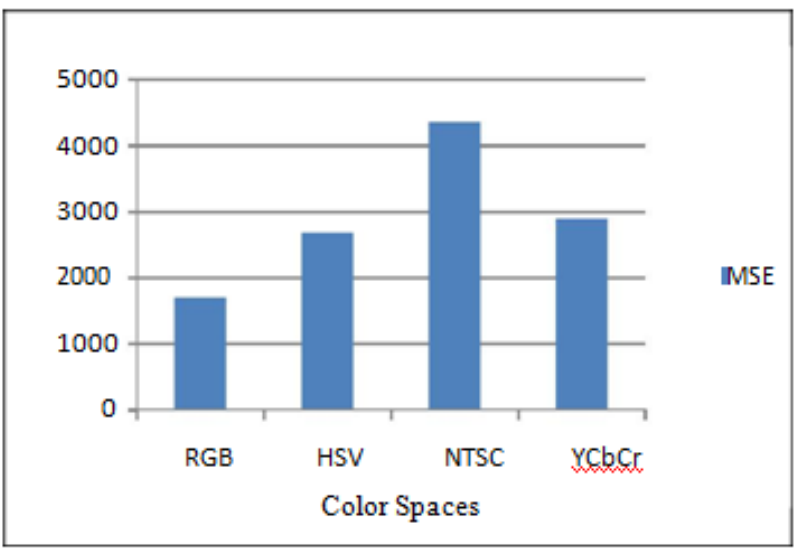

Fig 14: Comparison of results based on MSE

Sign Language Recognition is essential for the communication among hearing impaired people and other people. The paper focuses on analysing the results among color spaces and take decision based on them. These techniques are tested on a database of 156 images and results are analyzed on the basis of the results obtained on sample and sign images. Through their observations, we conclude that comparatively good results are obtained on RGB color space. This is merely the first step towards implementation of effective gesture recognition system. Further the project will be extended to recognize the detected gestures. Future Scope for this project will be focused on gestures with different background and different sign language.

\section{REFERENCES}

[1] Dr. Sudeep D. Thepade, Arati Narkhede Priti Kelvekar "Novel Technique for Background Removal from Sign Images for Sign Language Recognition System" International Journal of Computer Applications, (0975 8887) Volume 78 - No.10, September 2013.

[2] Dr.Sudeep.D.Thepade, Ms.Arati Narkhede, Ms.Priti Kelvekar, Ms.Gandhali Kulkarni and Ms.Seema Tathe, “ Sign Language Recognition using Color Means of Gradient Slope Magnitude Edge Images" published at International Conference on Intelligent Systems and Signal Processing (ISSP2013), 978-81-909376-6-5 2013IEEE.

[3] Rehanullah Khan, Zeeshan Khan , Muhammad Aamir, Syed Qasim Sattar, Sarhad University of Science and Information Technology,Peshawar,"Static Filtered Skin Detection" published at, IJCSI International Journal of Computer Science Issues, Vol. 9, Issue 2, No 3, March 2012 ISSN (Online):1694-0814.

[4] Ms.Prajakta M.Patil Prof.Y.M.Patil Assistant Professor of $\mathrm{E}$ and $\mathrm{TC}$,Engineering, Professor of Electronics Engineering, ADCET, Ashta, India KIT,Kolhapur, India, "Robust Skin Colour Detection And Tracking Algorithm" published at International Journal of Engineering Research and Technology (IJERT) Vol. 1 Issue 8, October 2012.

[5] http://lifeprint.com/as1101/topics/wallpaper1.html

[6] www.pics4.this-pic.com

[7] Dr. H B Kekre, Sudeep D Thepade and Adib Parkar.Article:Storage of Colour Information in a Greyscale Image using Haar Wavelets and Various Colour Spaces.International Journal of Computer Applications 6(7):18-24, September 2010. Published By Foundation of Computer Science. 
[8] H. B. Kekre, Sudeep D. Thepade"Color Based Image Retrieval using Amendment of Block Truncation Coding with YCbCr Color Space" at International Journal of Imaging(ISSN-0974-0627).

[10] Nishu, Sunil Agarwal, "Quantifying the Defect Visibility in Digital Images by Proper Color Space Selection" at International Journal of Engineering Research and Applications Vol. 2, Issue 3, May-Jun 2012, pp.1764-1767.

[11] HB Kekre, Sudeep D Thepade, "Image Retrieval using augmented block truncation coding technique" at
Proceedings of the International Conference on Advances in Computing, Communication and Control.

[12] HB Kekre, Sudeep D Thepade, Varun K Banura, "Amelioration of Walsh-Hadamard Texture Patterns based Image Retrieval using HSV Color Space" at International Journal of Computer Science and Information Security.

[13] V.K. Banura H.B.Kekre, Dr. Sudeep D.Thepade, Dr. T.Sarode, "Performance Comparison of Image Retrieval using KFCG with Assorted Pixel Window Sizes in RGB and LUV Color Spaces" at International Journal of Electronics and Communication Technologies. 